\title{
Input Impedance and Current Feedforward Control of Single-Phase Boost PFC Converters
}

\author{
Sungmin Park ${ }^{*}$, Sung-Yeul Park ${ }^{\dagger}$, and Ali M. Bazzi ${ }^{*}$ \\ ${ }^{* \dagger}$ Department of Electrical and Computer Engineering, University of Connecticut, Storrs, Connecticut, U.S.A.
}

\begin{abstract}
The combination of voltage feedforward and feedback control is a conventional approach for correcting the power factor in single-phase ac-dc boost converters. The feedback duty ratio increases significantly with an increase of the line frequency and input inductance. Therefore, the performance of the conventional approach is highly dependent on the bandwidth of the feedback controller. As a result, the input power quality can be significantly exacerbated due to uncompensated duty ratios if the feedback controller is limited. This paper proposes an input impedance and current feedforward control method to reduce the control portion of the feedback controller. The findings in this paper are 1) the theoretical derivation and analysis of variations of line frequency and input inductance on a power factor correction approach, 2) guaranteed consistent performance in a wide range of conditions, and 3) that a low switching frequency can be utilized by the proposed method. A MATLAB/Simulink model and a $1.2 \mathrm{~kW}$ dual boost converter are built to demonstrate the effectiveness of the proposed method.
\end{abstract}

Key words: Ac-dc boost converter, Dual boost PFC converter, Feedforward control, Power Factor Correction (PFC)

\section{INTRODUCTION}

Power factor correction (PFC) technology has been widely applied in industrial and commercial products for ac-dc power conversion. Therefore, PFC technology is considered to be a mature discipline in terms of high efficiency and high power quality. This was achieved through advanced circuit topologies and control algorithms due to an immense amount of research [1]-[3]. The demand for higher efficiency and higher quality power is always a major concern for manufacturers.

In general, when converters are designed, there are tradeoffs between high efficiency and high power quality in terms of the switching frequency. A higher switching frequency synchronized with the sampling rate yields a lower total harmonic distortion (THD) because of the high bandwidth of the current-loop compensator. However, the efficiency can be reduced due to the increased switching losses, and vice versa. Reducing the switching losses while using a high switching frequency is fairly restricted by the

Manuscript received Aug. 20, 2014; accepted Dec. 26, 2014

Recommended for publication by Associate Editor Yan Xing.

${ }^{\dagger}$ Corresponding Author: supark@engr.uconn.edu

Tel:+1-860-486-4822, Fax:+1-860-486-2447,University of Connecticut

* Department of Electrical and Computer Engineering, University of Connecticut, U.S.A. electrical characteristics of semiconductors unless a new paradigm of power devices, such as silicon carbide devices, is considered [4]. Therefore, it is desirable to use the lowest possible switching frequency to increase the converter efficiency if the low bandwidth current-loop issue is to be circumvented. With the growing trend toward employing digital control for the sophisticated functions of products, it is preferable to implement this solution on a digital platform.

A simple control method through a nonlinear-carrier (NLC) that allows operation in the continuous conduction mode (CCM) without a source voltage sensor has been described in [5]. This approach is more suitable in analog implementations. Extended versions of the NLC for digital implementation, digital nonlinear carrier (DNLC) methods, have been reported in [6]-[8]. [6] and [7] used only an instantaneous input current and a proportional gain for controlling the dc-link voltage. A partial switching operation is used to reduce the switching losses in [6], and a low-cost solution using a low-resolution DPWM and low-resolution A/D converters is used in [7]. In addition, a DNLC with a variable slope ramp has been presented in [8] to reduce the complexity of integrated circuit realization. However, these methods excluded current loop compensation. In addition, they may not guarantee stable operation during transients, or protect devices and circuits from overcurrent during unexpected fault 
conditions.

Predictive current control methods for single-phase ac-dc boost converters have been presented in [9]-[11]. The desired next duty ratio to yield the current reference can be predicted through calculations based on the sensed or observed state if the mathematical model of the system is known. However, since the performance is highly dependent on the circuit parameters, which may be sensitive to temperature changes, it requires accurate estimation of the parameter values under uncertainties.

Leading-phase admittance cancellation (LPAC) techniques have been presented in [12] and [13] to improve the current control and to eliminate the leading-phase of line current through a properly designed admittance compensator without increasing the bandwidth of the current-loop compensator. Nevertheless, these methods only considered the leading phase admittance, and the complexity of designing this admittance compensator makes it less attractive.

Attempts to eliminate the zero-crossing distortion of input current through voltage feedforward control methods have been presented in [14]-[18]. A voltage feedforward duty ratio signal is adopted to produce an average switch voltage over a switching cycle, which reduces the control proportions of a regular feedback current-loop compensator [14]. The methods in [14] and [15] employ a full feedforward control signal consisting of the instantaneous line voltage and the derivative of the reference current. Sensorless control methods for PFC without input voltage and current sensors have been presented in [16] and [17] and a plug-in repetitive control scheme was investigated in [18] under the voltage feedforward control method. However, these methods may not accomplish a unity power factor due to lagging-phase admittances if the current-loop compensator does not have enough bandwidth. Recently, many papers have focued on low-cost PFC solutions through the use of low-performance controllers and the elimination of sensors such as current [19]-[21] and voltage sensors [22], [23] rather than improving the input power quality.

Most of the control methods reported in the literature for improving input current quality have focused on compen sation methods for the leading-phase effects with a well-regulated current compensator in spite of the advent of lagging-phase admittances in some conditions where a low switching frequency is used in a high-line frequency.

This paper proposes an input impedance and current (IIC) feedforward control method. It employs a simple modification of the conventional voltage feedforward control method which is comonly used in PFC applications. A dual boost PFC converter is utilized here to reduce conduction losses [16], [24]-[26]. The proposed method also reduces switching losses with a low bandwidth current-loop compensator. By applying the proposed IIC feedforward control scheme, the feedforward signal can cancel

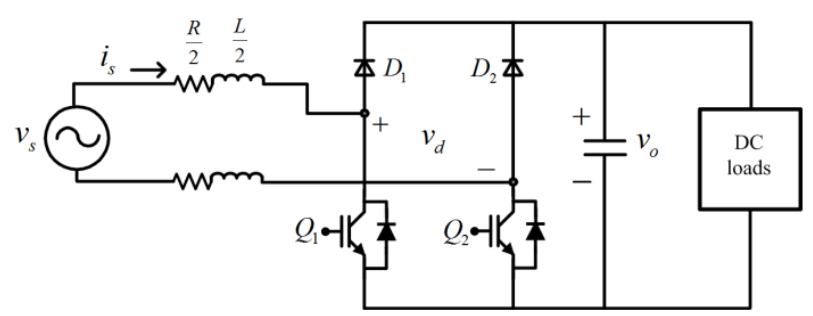

Fig. 1. Dual boost PFC converter configuration.

undesirable leading phase admittances as well as lagging phase admittances, with a low bandwidth current-loop compensator. Thus, this method provides more applicable solutions for ac-dc boost converters in low switching sampling frequency and high-line frequency applications.

This paper starts with a brief review of the conventional voltage feedforward control method in Section II. The proposed IIC feedforward control scheme and small-signal input admittances are derived and presented in Section III. Detailed comparisons of various control methods are carried out and briefly discussed in Section IV. MATLAB/Simulink simulation results comparing the performances of three control methods are shown in Section V. Experimental verification of the proposed approach is presented in section VI. Finally, Section VII concludes the paper.

\section{VOLTAGE FEEDFORWARD CONTROL}

The conventional voltage feedforward control method discussed in [14]-[18] and has been used as a standard practice to improve the input power quality of converters in digital implementations. This section explains the main features and limitations of the conventional feedforward method under a limited-bandwidth current compensator.

\section{A. Derivation of the Conventional Voltage Feedforward Control}

From the dual boost converter, as shown in Fig. 1, with an input inductor, $L$, and its parasitic resistor, $R$, Kirchhoff's voltage law with the source voltage, $v_{s}$, the switch voltage, $v_{d}$ and the input line current, $i_{s}$, yields:

$$
v_{s}=R i_{s}+L \frac{d i_{S}}{d t}+v_{d}
$$

where $v_{S}$ is the instantaneous value of the source voltage expressed as $V_{M} \sin (\omega t)$. The switch voltage is always a major factor in determining the waveform of the input current. In other words, when producing a sinusoidal input current, the switch voltage has to emulate the source voltage identically, with the exact phase difference due to the input impedance. The average switch voltage over a switching cycle at a positive source voltage in the CCM, can be expressed as:

$$
v_{d}=(1-d) v_{o}
$$

where, $d$ is the average on-time duty ratio of the switches, 
and $v_{o}$ is the dc output voltage shown in Fig. 1. By combining (1) and (2), the equation can be rewritten as:

$$
v_{s}=R i_{s}+L \frac{d i_{s}}{d t}+(1-d) v_{o}
$$

By rearranging (3) in terms of $d$, the duty ratio equation can be obtained as:

$$
d=\underbrace{\frac{1}{v_{o}}\left(R i_{s}+L \frac{d i_{s}}{d t}\right)}_{d_{F B}}+\underbrace{\left(1-\frac{v_{s}}{v_{o}}\right)}_{d_{F F}}
$$

Theoretically, the duty ratio in (4) should be generated for an ideal switch voltage as accurately as possible. This can be accomplished through adequate converter compensators to yield a pure sinusoidal input current. Under the assumption that the phase difference due to the input impedance is relatively small, the two voltage waveforms should be almost identical [14]. However, this assumption may lead to lagging-phase shift problems of the input current if a large inductance and a low bandwidth current-loop compensator are employed at a high line frequency. This will be elaborated upon later in the paper.

\section{B. Problems in Low Switching and High-line Frequency Applications}

The duty ratio, $d$ of the system in (4) consists of the feedback duty ratio $d_{F B}$ and the feedforward duty ratio $d_{F F}$. $d_{F B}$ contributes to the generation of the exact phase difference between the source voltage and the average switch voltage, which can be obtained through a simple proportional-integral (PI) compensator. $d_{F F}$ produces the inverse of the source voltage waveform as the average switch voltage. If the feedforward controller is not used, the compensator is heavily burdened with producing the total duty value $\left(d_{F B}+d_{F F}\right)$ in (4) and the system will require a high bandwidth compensator.

Fig. 2 asserts that the phase difference between $v_{s}$ and $v_{d}$ (not shown but represented by $d$ as shown in (2) and (4)), becomes significantly larger when the boost inductance and line frequency increase. $d_{F B}$ needs to be generated more accurately when the feedback controller's contribution increases.

If the limited current-loop compensator yielding $d_{F B}$ compensates the phase difference incompletely, then this condition causes a lagging-phase input admittance. This may result in an undesired input current distortion and a displacement of the phase. The authors of [12]-[15] focused mainly on ameliorating distortions of the input admittances in the leading-phase region caused by the dynamics of the current-loop. This does not directly address the issue of the lagging phase caused by the boost inductor and the limited bandwidth current-loop compensator. The lagging region addressed in [12]-[15], [18] was located in a high frequency range from $3 \mathrm{kHz}$ to $10 \mathrm{kHz}$, and did not cause an issue due to the high bandwidth of the current-loop compensator and

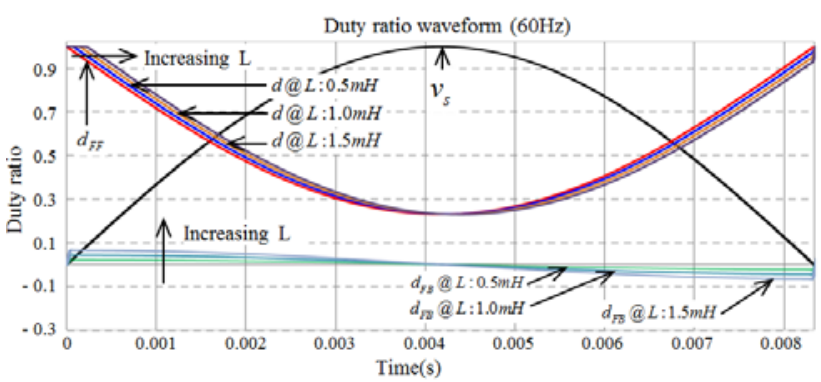

(a)

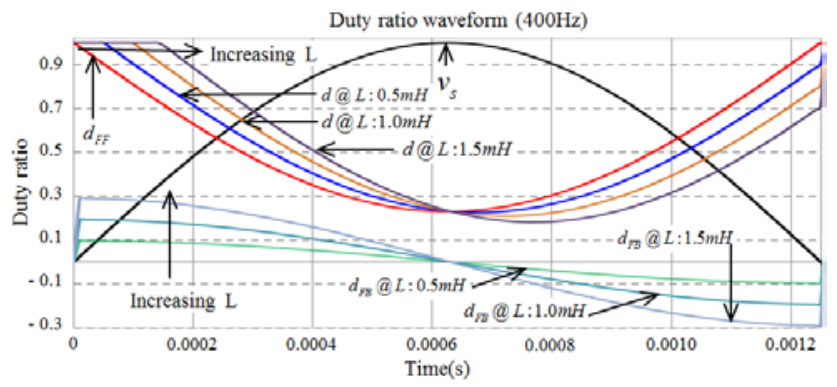

(b)

Fig. 2. Duty waveforms of feedback and feedforward controllers. (a) Line frequency $(60 \mathrm{~Hz})$. (b) Line frequency $(400 \mathrm{~Hz})$.

the low input inductance.

In the conventional voltage feedforward scheme, (4) indicates that $d_{F F}$ does not exhibit compensator terms to reduce the lagging-phase effects. It remains unchanged regardless of the leading or lagging input current because $d_{F F}$ is related to the input and output voltages. The conventional scheme depends only on the performance of the current-loop compensator to eliminate the lagging-phase effects. As a result, the converter encounters a non-unity power factor if the bandwidth of the current-loop compensator is limited.

\section{INPUT IMPEDANCE AND CURRENT FEEDFORWARD CONTROL}

\section{A. Derivation of the IIC Feedforward Control}

The proposed IIC feedforward control method is based on surveys of the phase information of the input current to determine whether it is lagging or leading with respect to the source voltage. Combining a simple current control law and the conventional voltage feedforward control duty, a new feedforward control signal can be expressed under the assumption that the power factor value is unity [6], [7], [27], [28].

$$
d_{I I C}=1-\frac{v_{s}}{v_{o}}=1-\frac{1}{G_{e} \cdot v_{o}} \cdot i_{s}=1-\frac{v_{s, r m s}^{2}}{P_{i n} \cdot v_{o}} \cdot i_{s}
$$

where, $\mathrm{G}_{e}=\frac{i_{s}}{v_{s}}=\frac{P_{i n}}{v_{s, r m s}^{2}}$

$G_{e}$ is the emulated input admittance, $v_{s, r m s}$ is the $R M S$ value of the source voltage, and $P_{i n}$ is the input power of the PFC rectifying stage. Furthermore, the input power can be 
expressed in terms of the RMS values of the source voltage and input current $i_{s, r m s}$ as:

$$
P_{i n}=v_{s, r m s} \cdot i_{s, r m s}
$$

By combining (5) and (6), the proposed IIC feedforward duty equation can be obtained as:

$$
d_{I I C}=1-\frac{v_{s, r m s}}{i_{s, r m s} \cdot v_{o}} \cdot i_{s}
$$

To exemplify how significantly the proposed feedforward controller reduces the control portion of the feedback controller, the duty ratio equations can be compared in the Laplace domain. Taking Laplace transforms of the source voltage and input current yields:

$$
\begin{aligned}
& v_{s}(s)=V_{s} \frac{\omega}{s^{2}+\omega^{2}} \\
& i_{s}(s)=I_{s} \frac{\omega \cos \phi+s \sin \phi}{s^{2}+\omega^{2}}
\end{aligned}
$$

where, $V_{s}$ and $I_{s}$ are the peak magnitude values, $\omega$ is the line angular frequency, and $\phi$ is the phase difference between the source voltage and the input current. By using (4), (8) and (9), the duty ratio using the conventional voltage feedforward term can be written in (10). In a similar manner, the duty ratio using the proposed IIC feedforward term can be written in (11). The feedback duty portions of the total duty for the two feedforward methods can be defined as:

$$
\begin{gathered}
\rho_{V F}(s)=\frac{d_{F B}(s)}{d_{F B}(s)+d_{F F}(s)} \\
\rho_{I I C}(s)=\frac{d_{F B}(s)}{d_{F B}(s)+d_{I I C}(s)}
\end{gathered}
$$

where $\rho_{V F}(s)$ and $\rho_{\text {IIC }}(s)$ are the feedback duty portions of the total duty with the conventional voltage feedforward and the proposed IIC feedforward, respectively. By using (10)-(13), (12) and (13) can be rewritten as (14) and (15). If a high-bandwidth current-loop compensator is implemented, the phase difference $\phi$ is zero. As a result, both (14) and (15) are identical. Otherwise, $\phi$ is nonzero and both (14) and (15) behave differently.

Fig. 3(a) shows a Bode plot generated at $\phi=0^{\circ}$ for (14) and (15), which are the same when $\phi=0^{\circ}$. The feedback duty portion at a line frequency of $60 \mathrm{~Hz}$ increases from $2 \%$ to $5 \%$ as the boost inductance increases from $0.5 \mathrm{mH}$ to $1.5 \mathrm{mH}$,

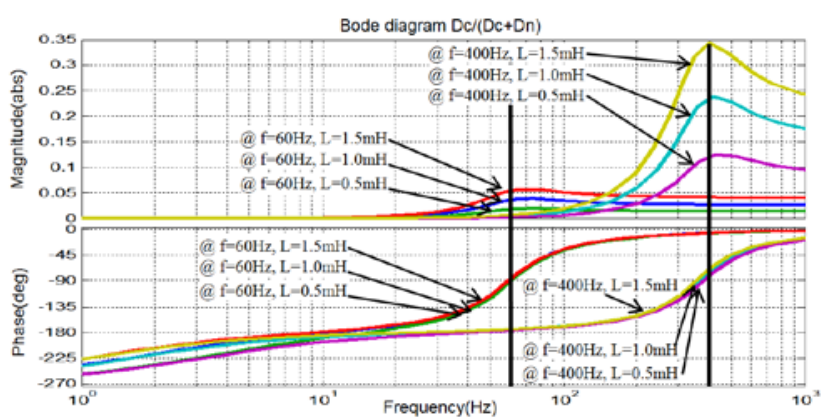

(a)

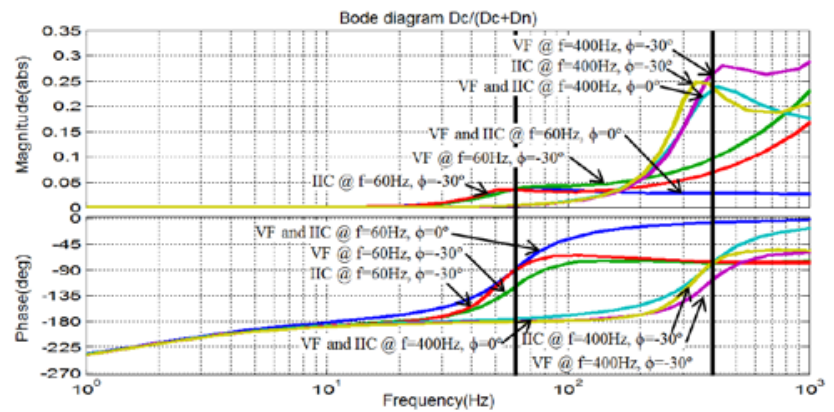

(b)

Fig. 3. Feedback duty portion of the total duty. (a) $\phi=0^{\circ}$ with different inductor values. (b) $\phi=-30^{\circ}$ with $1.0 \mathrm{mH}$ inductor and $\phi=0^{\circ}$ as reference value.

whereas this value at $400 \mathrm{~Hz}$ increases from $13 \%$ to $34 \%$. In reference to Fig. 2, the phase shift of the feedback duty portion is almost $90^{\circ}$ at $60 \mathrm{~Hz}$, and it is less than $90^{\circ}$ at $400 \mathrm{~Hz}$. Fig. 3(b) shows a reference value from Fig. 3(a) at $\phi=0^{\circ}$ and $\phi=-30^{\circ}$ for (14) and (15) at $60 \mathrm{~Hz}$ and $400 \mathrm{~Hz}$. It is important to note the following characteristics in Fig. 3 (b): 1) The phase of (15) in the proposed IIC feedforward method remains unchanged with the phase of the reference. However, the phase of (14) in the conventional voltage feedforward method becomes greater than the ideal value; and 2) The magnitude of (15) representing the feedback duty portion to the total duty is lower than that of (14), which can be distinguished at $400 \mathrm{~Hz}$. As a result, the proposed method can reduce the control portion of the compensator when compared to the conventional method. This indicates that the proposed method is less dependent on the performance of its current-loop compensator.

$$
\begin{gathered}
d_{F B}(s)+d_{F F}(s)=\frac{\left(I_{s} L \sin \phi\right) s^{3}+\left(v_{o}+I_{s} R \sin \phi+I_{s} \omega L \cos \phi\right) s^{2}+\left(I_{s} \omega R \cos \phi-V_{s} \omega\right) s+v_{o} \omega^{2}}{v_{o} s^{3}+\left(v_{o} \omega^{2}\right) s} \\
d_{F B}(s)+d_{I I C}(s)=\frac{\left(I_{s} L \sin \phi\right) s^{3}+\left(v_{o}-V_{s} \sin \phi+I_{s} R \sin \phi+I_{s} \omega L \cos \phi\right) s^{2}+\left(I_{s} \omega R \cos \phi-V_{s} \omega \cos \phi\right) s+v_{o} \omega^{2}}{v_{o} s^{3}+\left(v_{o} \omega^{2}\right) s} \\
\rho_{V F}(s)=\frac{\left(I_{s} L \sin \phi\right) s^{3}+\left(I_{s} R \sin \phi+I_{s} \omega L \cos \phi\right) s^{2}+\left(I_{s} \omega R \cos \phi\right) s}{\left(I_{s} L \sin \phi\right) s^{3}+\left(v_{o}+I_{s} R \sin \phi+I_{s} \omega L \cos \phi\right) s^{2}+\left(I_{s} \omega R \cos \phi-V_{s} \omega\right) s+v_{o} \omega^{2}} \\
\rho_{I I C}(s)=\frac{\left(I_{s} L \sin \phi\right) s^{3}+\left(I_{s} R \sin \phi+I_{s} \omega L \cos \phi\right) s^{2}+\left(I_{s} \omega R \cos \phi\right) s}{\left(I_{s} L \sin \phi\right) s^{3}+\left(v_{o}-V_{s} \sin \phi+I_{s} R \sin \phi+I_{s} \omega L \cos \phi\right) s^{2}+\left(I_{s} \omega R \cos \phi-V_{s} \omega \cos \phi\right) s+v_{o} \omega^{2}}
\end{gathered}
$$




\section{B. Input Admittances for Three Control Methods}

The benefits of the proposed IIC feedforward controller can be also verified by analyzing the input admittances in the frequency domain. Recent studies [14], [15], [27], [28] have helped to predict the behaviors of the input admittances for designing control algorithms. Fig. 4 depicts a control block diagram including the regular current-loop compensator and the feedforward controller.

Fig. 5 describes three control methods; "without any feedforward," "with the conventional voltage feedforward," and "with the proposed IIC feedforward." By employing linearized input admittances, the input current quality can be assessed and the harmonic distortion can be predicted in different input frequency ranges. Using (2) in (1) and applying small perturbations, the response of the ac-dc boost converter can be expressed as:

$$
\hat{v}_{s}(s)=(s L+R) \hat{i}_{s}(s)+(1-D) \hat{v}_{o}(s)+V_{o} \hat{d}(s)
$$

where the capital letters are the values of system variables at a steady-state operating point, and the hatted lowercase letters are small perturbations in the steady state.

The linearized versions of the feedback duty $d_{F B}(s)$, the voltage feedforward duty $d_{F F}(s)$, and the proposed IIC feedforward duty $d_{\text {IIC }}(s)$ can be obtained as:

$$
\begin{gathered}
\hat{d}_{F B}(s)=\left(\frac{I_{\text {OREF }}}{V_{M}} \hat{v}_{s}(s)-\hat{i}_{s}(s)\right) G_{c}(s) \\
\hat{d}_{F F}(s)=\frac{V_{s}}{V_{o}} \hat{v}_{o}(s)-\frac{1}{V_{o}} \hat{v}_{s}(s) \\
\hat{d}_{I I C}(s)=\frac{1}{G_{e}}\left(\frac{I_{s}}{V_{o}} \hat{v}_{o}(s)-\frac{1}{V_{o}} \hat{i}_{s}(s)\right)
\end{gathered}
$$

From Fig. 5, the small-signal transfer functions of the final output duties of the three control methods can be obtained in (20)-(22), where $\hat{d}_{c c}(\mathrm{~s}), \hat{d}_{f f}$ (s) and $\hat{d}_{i i c}$ (s) are the small-signal duty transfer functions of the ac-dc boost converter with the regular current-loop compensator, the conventional voltage feedforward duty, and the proposed IIC method, respectively.

The following derivations are performed under the assumption that the output of the dc voltage compensator is constant and the delay of the transducers is small when calculating the input impedance in the high-frequency region. Using (20)-(22) in the duty term of (16), to eliminate the duty terms, the small-signal input admittances of the ac-dc

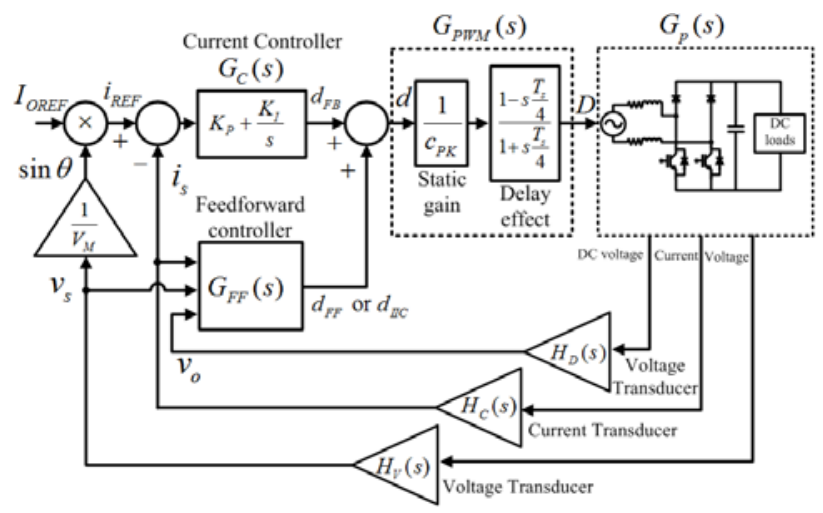

Fig. 4. Control block diagram with feedforward controllers.

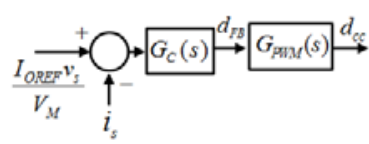

(a)

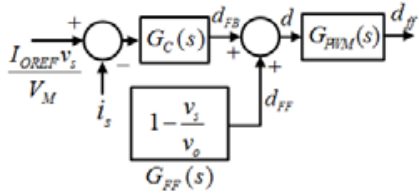

(b)

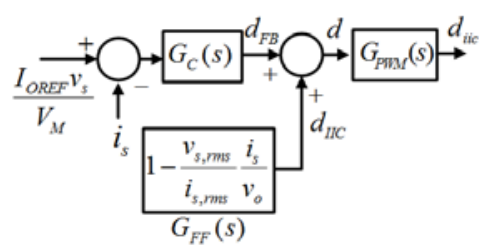

(c)

Fig. 5. Three control methods. (a) Without any feedforward. (b) With the conventional voltage feedforward. (c) With the proposed IIC feedforward.

converter using the three control strategies can be obtained as:

$$
\begin{gathered}
G_{c c}(s)=\frac{\hat{i}_{s}(s)}{\hat{v}_{s}(s)}=\frac{1+\frac{I_{\text {OREF }} V_{o} G_{c}(s) G_{P W M}(s)}{V_{M}}}{s L+R+V_{o} G_{c}(s) G_{P W M}(s)} \\
G_{f f}(s)=\frac{1-G_{P W M}(s)+\frac{I_{O R E F} V_{o} G_{c}(s) G_{P W M}(s)}{V_{M}}}{s L+R+V_{o} G_{c}(s) G_{P W M}(s)} \\
G_{i i c}(s)=\frac{I_{\text {OREF }} V_{o} G_{c}(s) G_{P W M}(s)}{s L+R+V_{o} G_{c}(s) G_{P W M}(s)+\frac{V_{M}}{I_{O R E F}} G_{P W M}(s)}
\end{gathered}
$$


In (23)-(25), $G_{c c}(s), G_{f f}(s)$, and $G_{i i c}(s)$ are the small-signal input admittance transfer functions of the ac-dc boost converter with the regular current-loop compensator, the conventional voltage feedforward duty, and the proposed IIC feedforward method, respectively. Furthermore, if the delay influence of the PWM is negligible over the frequency ranges of interest and the static gain is unity, $G_{P W M}(s)$ can be modeled as a constant unity gain under the average current control. Hence, the small-signal input admittances can be approximated by:

$$
\begin{gathered}
G_{c c}(s) \approx \frac{1+G_{e} T(s)}{s L+R+T(s)} \\
G_{f f}(s) \approx \frac{G_{e} T(s)}{s L+R+T(s)} \\
G_{i i c}(s) \approx \frac{G_{e}\left(T(s)+1 / G_{e}\right)}{s L+R+\left(T(s)+1 / G_{e}\right)}
\end{gathered}
$$

where, $T(s)=V_{o} G_{c}(s) G_{P W M}(s), G_{e}=\frac{I_{O R E F}}{V_{M}}$

It can be observed in (26)-(28) that if it is assumed that the impedance of the boost inductors is negligible over the low frequency ranges of interest, $G_{c c}(s)$ approaches $1 / T(s)+G_{e}$, which is the leading-phase effect caused by the dynamics of the current-loop compensator [27]. $G_{f f}(s)$ and $G_{i i c}(s)$ both approach the constant $G_{e}$. This implies that they act in a purely resistive manner. For this reason, a higher quality input current can be obtained through feedforward control schemes.

\section{COMPARISON OF SMALL-SIGNAL INPUT ADMITTANCES}

In this section, the distortion and contribution factors are introduced to explain the effectiveness of the proposed IIC feedforward controller. The deviation in the actual impedance from the expected impedance is referred to as the distortion factor. The distortion factors of the input admittances for the aforementioned control methods are defined by:

$$
\begin{gathered}
A_{c c}(s)=\frac{G_{c c}(s)}{G_{e}}=\frac{1 / G_{e}+T(s)}{s L+R+T(s)} \\
A_{f f}(s)=\frac{G_{f f}(s)}{G_{e}}=\frac{T(s)}{s L+R+T(s)} \\
A_{i i c}(s)=\frac{G_{i i c}(s)}{G_{e}}=\frac{T(s)+1 / G_{e}}{s L+R+T(s)+1 / G_{e}}
\end{gathered}
$$

It can be observed that the three distortion factors in (29)-(31) are identical if the total input impedance $\left(1 / G_{e}\right)$ of the converters approaches zero. Similarly, if the inductance and parasitic resistance $(s L+R)$ of the boost inductor are ignored, as assumed in [14], the other two distortion factors for the feedforward controllers occur at unity and there is no distortion. However, the boost inductor impedance terms in the denominators of the distortion factors become

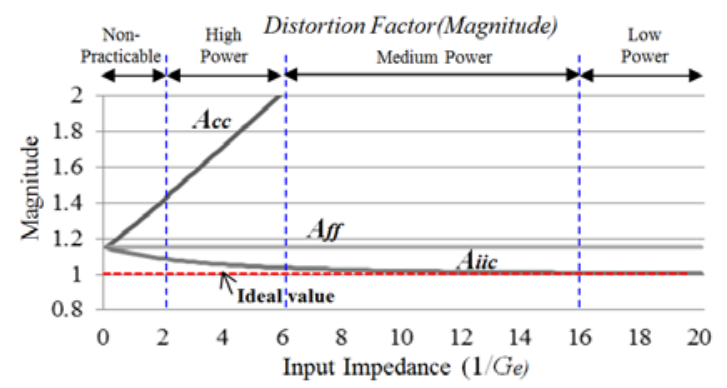

(a)

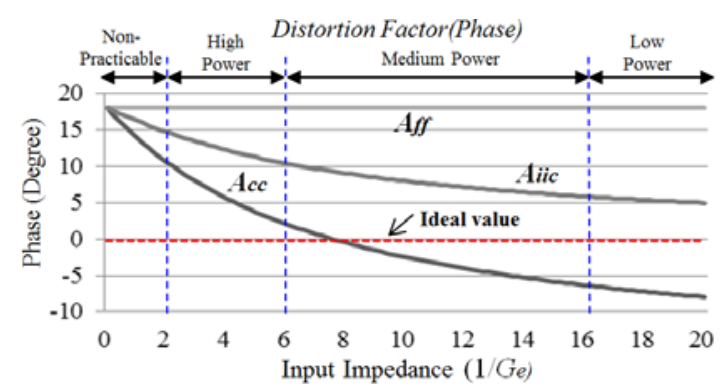

(b)

Fig. 6. Distortion factors for three control methods vs. input impedance. (a) Maginitude response. (b) Phase response.

overwhelming and force the distortion factors into a lagging-phase as the line frequency rises. In low switching frequency applications, the zero-phase crossover occurs at a lower frequency. Thus, the boost inductor impedance term is no longer negligible. The boost inductor impedance term should be eliminated by a high bandwidth current-loop compensator. Due to this phenomenon, the conventional voltage feed-forward control may not be a suitable approach when the current-loop compensator has a limited bandwidth.

Ideally, it is desirable to achieve a distortion factor magnitude that is close to unity at a zero phase difference, yielding an ideal power factor correction. Fig. 6 shows the magnitude and phase response of the distortion factors when the input impedance increases, the input frequency is $400 \mathrm{~Hz}$, and the bandwidth of the current-loop compensator is $1 \mathrm{kHz}$. It can be observed that the magnitude and phase of $A_{i i c}(s)$ approaches the ideal value as the input impedance increases. Meanwhile $A_{f f}(s)$ has some deviation from the ideal value due to the unsatisfied current-loop compensator regardless of the input impedance. Fig. 7 shows Bode plots of the distortion factors in the frequency domain. By employing the proposed method, the input admittance is more constant and the lag is reduced.

As another comparison factor, the contribution factors are introduced to describe how the proposed IIC feedforward method can effectively reduce undesired input admittance.

$$
\begin{gathered}
K_{f f}(s)=\frac{G_{f f}(s)}{G_{c c}(s)}=\frac{G_{e} T(s)}{1+G_{e} T(s)} \\
K_{i i c}(s)=\frac{G_{i i c}(s)}{G_{c c}(s)}=\frac{s L+R+T(s)}{s L+R+T(s)+1 / G_{e}}
\end{gathered}
$$




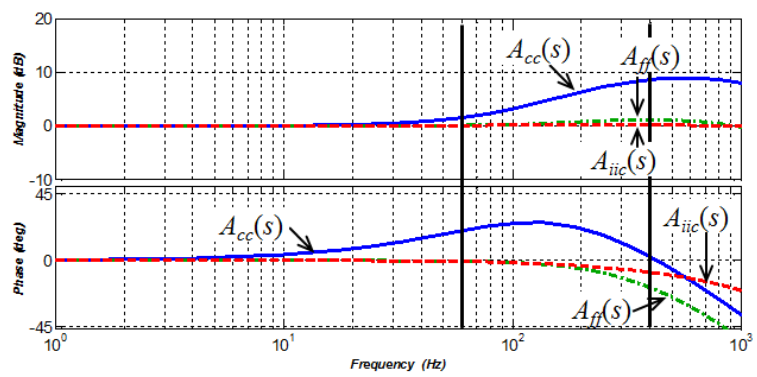

Fig. 7. Bode plots of distortion factor when the bandwidth of compensator is $1 \mathrm{kHz}$ at $G_{e}=1 / 10.3$.

Fig. 8 shows Bode plots of the contribution factors $K_{f f}(s)$ and $K_{i i c}(s)$ to the input admittance $G_{c c}(s)$. It should be noted that the phase of $G_{c c}(s)$ is changed from the leading phase to the lagging phase at around $450 \mathrm{~Hz}$. Thus, the lagging input admittance appears in the lower frequency range as the bandwidth of the compensator becomes more limited. It should be noted that these controllers show similar features for eliminating the undesired input admittance of $G_{c c}(s)$ below $100 \mathrm{~Hz}$. However, $K_{i i c}(s)$ cancels the distorted and displaced input admittance more properly than $K_{f f}(s)$. In other words, the proposed IIC feedforward controller can compensate for an inductive input admittance as the line frequency increases, and a capacitive input admittance as the line frequency decreases. The superiority of the proposed method over the conventional one becomes obvious as the performance of the current-loop compensator becomes worse.

In addition, it can be seen in (32) and (33) that the contribution factor of the proposed IIC feedforward method includes inductor impedance in both the numerator and the denominator. From this, it can be inferred that $G_{i i c}(s)$ is less sensitive to inductance variations. Fig. 9 shows $G_{f f}(s)$ and $G_{i i c}(s)$ under boost inductance $(L)$ variations to compare their sensitivity and uncertainties. As expected, the deviation of $G_{f f}(s)$ is significant under inductance variations from $50 \%$ to $200 \%$, as shown in Fig. 9(a), while $G_{\text {iic }}(s)$ is less sensitive as shown in Fig. 9(b).

\section{SIMULATION RESULTS}

The dual boost PFC converter is simulated in the MATLAB/Simulink environment with the major parameters as follows: $L=0.9 \mathrm{mH}, C_{d c}=2040 \mu \mathrm{F}, v_{o}=200 \mathrm{~V}$, and $v_{s, r m s}=$ $110 V_{r m s}$. In particular, a low switching frequency of $15 \mathrm{kHz}$ is used to investigate the effectiveness and performance of the proposed control. Meanwhile most PFC applications use a relatively high switching frequency in the range of $40 \mathrm{kHz} 130 \mathrm{kHz}$ [5], [7]-[9], [12], [14], [16]-[23], [25]-[27], and only two papers [6], [11] use $16 \mathrm{kHz}$ and $24 \mathrm{kHz}$, respectively, to validate their performance in low switching frequency applications. For an evaluation of the performance, the converter operation under three control strategies with a 1 $\mathrm{kHz}$ bandwidth of the current compensator was simulated: 1)

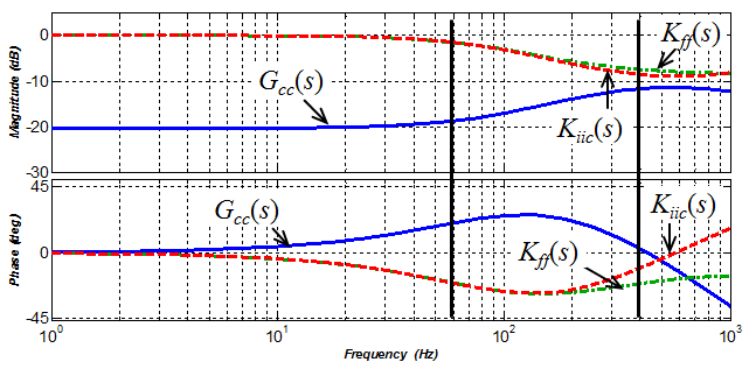

Fig. 8. Bode plots for input admittance of converter without feedforward controller and the contribution terms of feedforward controllers when the bandwidth of current loop controller is 1 $\mathrm{kHz}$.

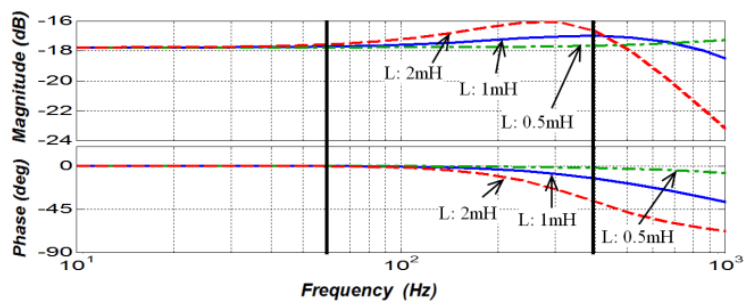

(a)

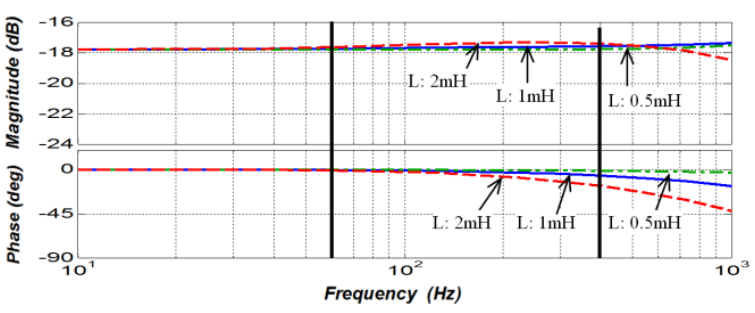

(b)

Fig. 9. Bode plots for input admittance under inductance variations at $G_{e}=1 / 10.3$. (a) the conventional voltage feedforward. (b) the proposed IIC feedforward.

without employing any feed-forward controllers, 2) with the conventional voltage feedforward control, and 3) with the proposed IIC feedforward control.

Fig. 10 compares the steady state input current waveforms obtained when the source voltage was $110 \mathrm{Vrms} / 60 \mathrm{~Hz}$. It is worth noting that with a band-limited compensator, the leading-phase effects and the zero-crossing distortions of the input current are observed at the nominal input frequency $(60 \mathrm{~Hz})$, as shown in Fig.10(a). However, these distortion factors completely disappear, as shown in Fig. 10(b) and Fig. 10(c), when the feedforward methods are applied. Similarly, at a high input frequency $(400 \mathrm{~Hz})$, the distortion and displacement factors of the input current are significantly worsened, as shown in Fig. 11(a) and Fig. 11(b), due to the lagging-phase effects. Meanwhile a significant reduction in terms of the displacement value has been achieved in the proposed controller when compared to the conventional voltage feedforward controller, as shown in Fig. 11(c). The simulation results demonstrate the proposed method's superiority in the time domain, and Fig. 7 displays this in the frequency domain. 


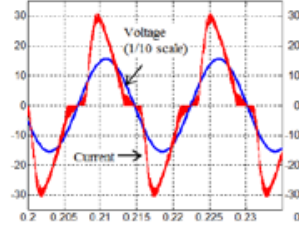

(a)

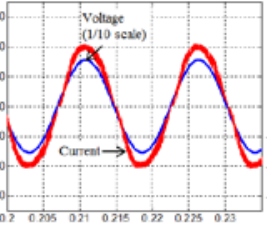

(b)

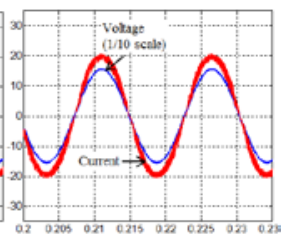

(c)
Fig. 10. Simulation results; line frequency: $60 \mathrm{~Hz}$. (a) without any feed-forward control (PF: 0.93, THD: 33.4\%). (b) the conventinal voltage feedforward (PF: 0.99, THD: 4.5\%). (c) the proposed IIC feedforward (PF: 1.0, THD: $2.1 \%$ ).

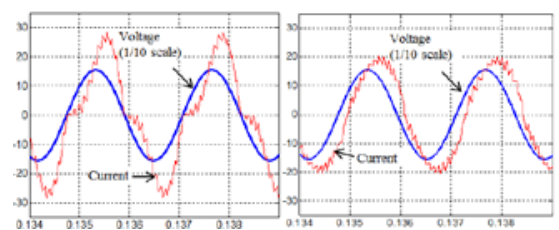

(a)

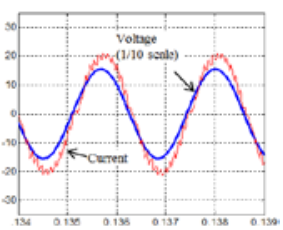

(c)
Fig. 11. Simulation results; line frequency: $400 \mathrm{~Hz}$. (a) without any feed-forward control (PF: 0.89, THD: 28.7\%). (b) the conventinal voltage feedforward (PF: 0.86, THD: 10.1\%). (c) the proposed IIC feedforward (PF: 0.98, THD:7.3\%).

\section{EXPERIMENTAL RESULTS}

Fig. 12 shows a prototype ac-dc and dc-dc converter for a battery charger. For the ac-dc converter, a single-phase dual boost converter based on a low-cost digital control was used to verify the proposed IIC feedforward control. Table I lists some of the important experimental values.

Fig. 13 shows experimental results comparing the performances of the conventional feedforward controller and the proposed IIC feedforward controller. Both of them have a $1 \mathrm{kHz}$ bandwidth current compensator. Fig. 13(a) and Fig. 13(b) show the input current and voltage at the nominal input frequency $(60 \mathrm{~Hz})$. When feedforward controllers are employed, an exceptionally high performance with a low distortion factor and a low displacement factor can be seen. However, the input current shown in Fig. 14(a), using the conventional feedforward controller, is displaced significantly at a high input frequency $(400 \mathrm{~Hz})$ due to the effect of uncompensated lagging-phase admittance. Meanwhile, the input current shown in Fig. 14(b), using the proposed IIC feedforward control, is less displaced and still has an acceptable PFC performance with a low bandwidth compensator which indicates a reduced ratio of the switching frequency to the input frequency.

The results for the THD and power factor between the two feedforward methods are compared in Table II. The proposed IIC feedforward control has superior performance. It results in a $17 \%$ improvement in the displacement factor and a $0.3 \%$ improvement in the distortion factor when the ac-dc boost converter has a limited-bandwidth current compensator at a $400 \mathrm{~Hz}$ line frequency. This can be explained with the analytical results in Fig. 7. The input admittances of the two

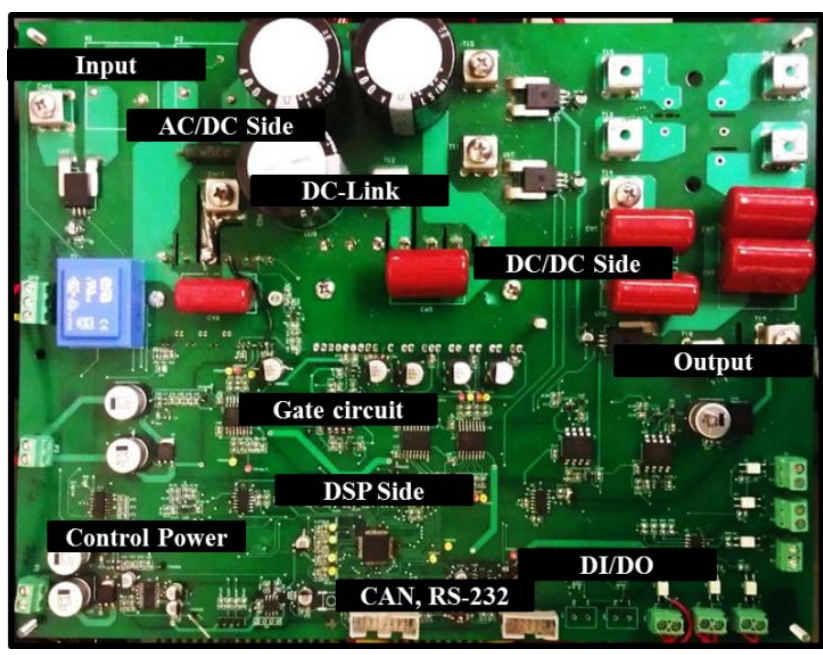

Fig. 12. Prototype dual boost PFC converter.

TABLE I

EXPERIMENTAL SETUP

\begin{tabular}{ll}
\hline \multicolumn{1}{c}{ System parameter } & \multicolumn{1}{c}{ Values } \\
\hline AC Source Voltage & $110 \mathrm{Vrms} / 60 \mathrm{~Hz}$ and 400Hz \\
Rated Power & $1.2 \mathrm{~kW}$ \\
Switching Freq. & $15 \mathrm{kHz}, 75 \mu$ s (Sampling time) \\
Boost Inductor & $0.9 \mathrm{mH}$ (split into two in series) \\
DSP & TMS320F28035(60MHz) \\
Power device & FPDB60PH60B (FAIRCHILD) \\
\hline
\end{tabular}

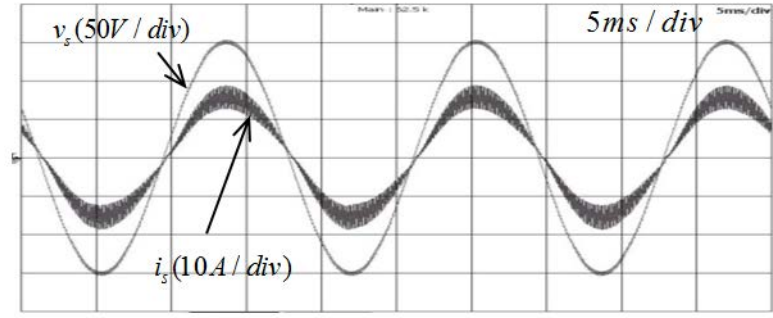

(a)

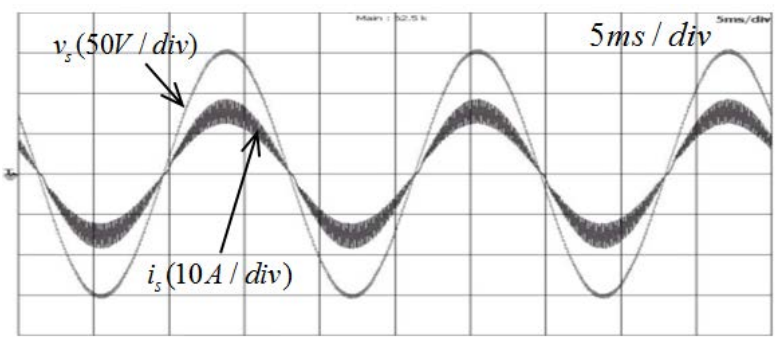

(b)

Fig. 13. Experimental results at line frequency $60 \mathrm{~Hz}$ - input voltage and input current. (a) Conventional feedforward control. (b) Proposed feedforward control.

feedforward control methods behave similarly with the input admittance at $60 \mathrm{~Hz}$. However, the proposed method has a distinguishably reduced lagging-phase at $400 \mathrm{~Hz}$ in the phase domain. When compared to the conventional method, the proposed method fares better in terms of the displacement 


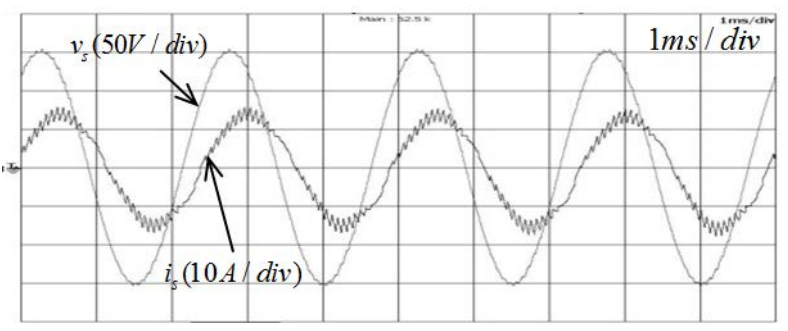

(a)

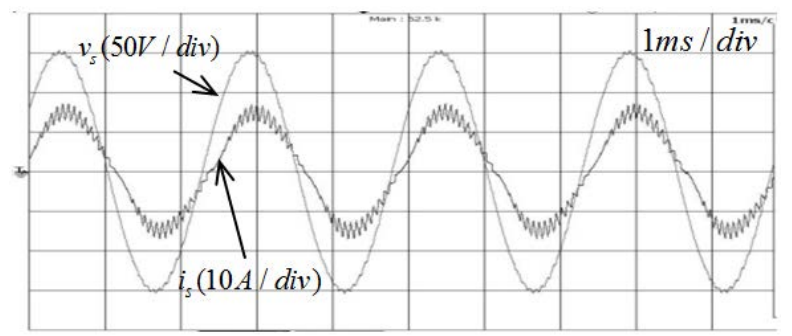

(b)

Fig. 14. Experimental results at line frequency $400 \mathrm{~Hz}$ - input voltage and input current. (a) Conventional feedforward control. (b) Proposed feedforward control.

TABLE II

SUMMARY OF EXPERIMENTAL RESULTS

\begin{tabular}{c|c|c|c|c}
\hline \multirow{2}{*}{ Method } & \multicolumn{2}{|c|}{$\begin{array}{c}\text { 60Hz line } \\
\text { frequency }\end{array}$} & \multicolumn{2}{c}{$\begin{array}{c}\text { 400Hz line } \\
\text { frequency }\end{array}$} \\
\cline { 2 - 5 } & THD & P.F & THD & P.F \\
\hline \hline Conventional & $4.0 \%$ & 1.0 & $5.3 \%$ & 0.80 \\
\hline Proposed IIC & $3.0 \%$ & 1.0 & $5.0 \%$ & 0.97 \\
\hline
\end{tabular}

factor rather than in the THD. The experimental results demonstrate that the proposed IIC feedforward controller is an enhanced solution for power factor correction at low switching/sampling frequency operation.

\section{CONCLUSION}

This paper has presented an input impedance and current (IIC) feedforward control method to solve the phase shift problems of the input current caused by lagging-phase admittances in low switching/sampling and high line frequency applications. The proposed method can reduce the undesired effects of input admittances over wide frequency ranges with a leading-lagging phase admittance cancellation. The effectiveness of the proposed method has been analyzed through small input admittances, distortion and contribution factors. Simulation and experimental results show that the input power quality is improved with the proposed IIC feedforward control, which supports the theoretical analysis. In addition, the proposed IIC feedforward method can be easily utilized with a simple modification of the existing voltage feedforward equation. Consequently, these features make the proposed IIC feedforward method an extremely good fit for digital implementation in ac-dc boost converters with a limited bandwidth.

\section{ACKNOWLEDGMENT}

This work is supported by the National Science Foundation (Award No. 1446157) received in the 2014. However, any opinions, findings, conclusions, or recommendations expressed herein are those of the authors and do not necessarily reflect the views of the National Science Foundation.

\section{REFERENCES}

[1] B. Singh, B. N. Singh, A. Chandra, K. Al-Haddad, A. Pandey, and D. P. Kothari, "A review of single-phase improved power quality ac-dc converters,” IEEE Trans. Ind. Electron., Vol. 50, No. 5, pp. 962-981, Oct. 2003.

[2] M. M. Jovanovic and Y. Jang, "State-of-the-art, single-phase, active power-factor-correction techniques for high-power applications - An overview," IEEE Trans. Ind. Electron., Vol. 52, No. 3, pp. 701-708, Jun. 2005.

[3] B. Singh and S. Singh, "Single-phase power factor controller topologies for permanent magnet brushless DC motor drives," IET Power Electron., Vol. 3, No. 2, pp. 147-175, Mar. 2010.

[4] J. G Kassakian and T. M. Jahns, "Evolving and emerging applications of power electronics in systems," IEEE J. Emerg. Sel. Topics Power Electron., Vol. 1, No. 2, pp. 47-58, Jun. 2013.

[5] D. Maksimović, Y. Jang, and R. W. Erickson, "Nonlinear-carrier control for high-power-factor boost rectifiers," IEEE Trans. Power Electron., Vol. 11, No. 4, pp. 578-584, Jul. 1996.

[6] Y. Notohara, T. Suzuki, T. Endo, H. Umeda, A. Okuyama, Y. Funayama, and K. Tamura, "Controlling power factor correction converter for single-phase AC power source without line voltage sensor," in Proc. Int. Power Elect. Conf. (IPEC), pp. 431-436, 2010.

[7] B. A. Mather and D. Maksimović, "A simple digital power-factor correction rectifier controller," IEEE Trans. Power Electron., Vol. 26, No. 1, pp. 9-19, Jan. 2011.

[8] J. Chiang, B. Liu, and S. Chen, “A simple implementation of nonlinear-carrier control for power factor correction rectifier with variable slope ramp onfield-programmable gate array,” IEEE Trans. Ind. Informat., Vol. 9, No. 3, pp. 1322-1329, Aug. 2013.

[9] J. Chen, A. Prodić, R. W. Erickson, and D. Maksimović, "Predictive digital current programmed control," IEEE Trans. Power Electron., Vol. 18, No. 1, Pt. 2, pp. 411-419, Jan. 2003.

[10] P. Athalye, D. Maksimovic, and R. W. Erickson, "DSP implementation of a single-cycle predictive current controller in a boost PFC rectifier," in Proc. IEEE Appl. Power Electron. Conf. Expo., pp. 837-842, 2005.

[11] L. Roggia, F. Beltrame, J. E. Baggio, and J. R. Pinheiro, "Digital current controllers applied to the boost power factor correction converter with load variation," IET Power Electron., Vol. 5, No. 5, pp.532-541, May 2012.

[12] K. P. Louganski and J.-S. Lai, "Current phase lead compensation in single-phase PFC boost converters with a reduced switching frequency to line frequency ratio," IEEE Trans. Power Electron., Vol. 22, No. 1, pp. 113-119, Jan. 2007. 
[13] S. Y. Park, C. L. Chen, J.-S. Lai, and S. R. Moon, "Admittance compensation in current loop control for a grid-tie LCL fuel cell inverter," IEEE Trans. Power Electron., Vol. 23, No. 4, pp. 1716-1723, Jul. 2008

[14] D. M. Van de Sype, K. De Gusseme, A. P. M. Van den Bossche, and J. A. Melkebeek, "Duty-ratio feedforward for digitally controlled boost PFC converters,” IEEE Trans. Ind. Electron., Vol. 52, No. 1, pp. 108-115, Feb. 2005.

[15] M. Chen and J. Sun, "Feedforward current control of boost single-phase PFC converters" IEEE Trans. Power Electron, Vol. 21, No. 2, pp. 338-345, Mar. 2006.

[16] G. G. Park, K. Y. Kwon, and T. W. Kim, "PFC dual boost converter based on input voltage estimation for DC inverter air conditioner," Journal of Power Electronics, Vol. 10, No. 3, pp. 293-299, May 2010.

[17] K. I. Hwu, H. W. Chen, and Y. T. Yau, "Fully digitalized implementation of PFC rectifier in CCM without ADC," IEEE Trans. Power Electron, Vol. 27, No. 9, pp. 4021-4029, Sep. 2012.

[18] Y. H. Cho and J. S. Lai, "Digital plug-in repetitive controller for single-phase bridgeless PFC converters," IEEE Trans. Power Electron., Vol. 28, No. 1, pp. 165-175, Jan. 2013.

[19] V. M. Lopez, F. J. Azcondo, A. de Castro, and R. Zane, "Universal digital controller for boost CCM power factor correction stages based on current rebuilding concept," IEEE Trans. Power Electron., Vol. 29, No. 7, pp. 3818-3829, Jul. 2014.

[20] A. Sanchez, A. de Castro, V. M. Lopez, F. J. Azcondo, and J. Garrido, "Single ADC digital PFC controller using precalculated duty cycles,” IEEE Trans. Power Electron., Vol. 29, No. 2, pp. 996-1005, Feb. 2014.

[21] M. Pahlevani, P. Shangzhi, S. Eren, A. Bakhshai, and P. Jain, "An adaptive nonlinear current observer for boost PFC AC/DC converters,” IEEE Trans. Ind. Electron., Vol. 61, No. 12, pp. 6720-6729, Dec. 2014.

[22] C. L. Nguyen, H. H. Lee, and T. W. Chun, "A simple grid voltage-sensorless control scheme for PFC boost converters," Journal of Power Electronics, Vol. 14, No. 4, pp. 712-721, Jul. 2014.

[23] H. J. Kim, G. S. Seo, B. H. Cho, and H. S. Choi, “A simple average current control with on-time doubler for multiphase CCM PFC converter,” IEEE Trans. Power Electron., Vol. 30, No. 3, pp. 1683-1693, Mar. 2015.

[24] D. M. Mitchell, “AC-DC Converter having an improved power factor,” U.S. Patent 4412277 A, Oct. 25, 1983.

[25] F. Musavi, W. Eberle, and W. G. Dunford, “A high-performance single-phase bridgeless interleaved PFC converter for plug-in hybrid electric vehicle battery chargers," IEEE Trans. Ind. Appl., Vol. 47, No. 4, pp. 1833-1843, Jul./Aug. 2011.

[26] L. Huber, Y. Jang, and M. M. Jovanovic, "Performance evaluation of bridgeless PFC boost rectifiers," IEEE Trans. Power Electron., Vol. 23, No. 3, pp. 1381-1390, May 2008.

[27] J. Sun, "Input impedance analysis of single-phase PFC converters," IEEE Trans. Power Electron., Vol. 20, No. 2, pp. 308-314, Mar. 2005.

[28] S. M. Park and S. Y. Park, "Input impedance and current feedforward control for leading-lagging phase admittance cancellation in the AC-DC boost converter," in Proc. IEEE Appl. Power Electron. Conf. Expo., pp. 1912-1919, 2013.

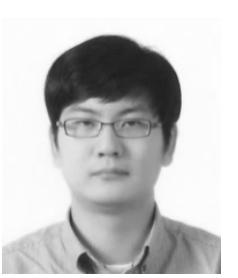

Sungmin Park received his B.S. degree in Electronics Engineering and his M.S. degree in Electrical Engineering from Korea University, Seoul, South Korea, in 2001 and 2003, respectively. He is presently working toward his Ph.D. degree in the Department of Electrical and Computer Engineering, University of Connecticut (UCONN), Storrs, CT, USA. From 2003 to 2008, he was a Senior Engineer at LG Electronics, Seoul, South Korea. From 2008 to 2010, he was with Samsung Heavy Industries, Suwon, South Korea. Since 2014, he has been a Senior Research Engineer at the United Technologies Research Center, East Hartford, CT, USA. His current research interests include the application of power electronics to home appliances and renewable energy systems.

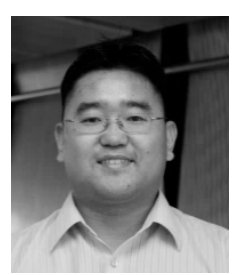

Sung-Yeul Park received his M.S. and Ph.D. degrees in Electrical and Computer Engineering from the Virginia Polytechnic Institute and State University (Virginia Tech), Blacksburg, VA, USA, in 2004 and 2009, respectively. From 2002 to 2004, he was a Graduate Research Assistant with the Center for Rapid Transit Systems, Virginia Tech. From 2004 to 2009, he was a Graduate Research Assistant with the Future Energy Electronics Center, Virginia Tech. He is presently an Assistant Professor with the Department of Electrical and Computer Engineering, University of Connecticut (UCONN), Storrs, CT, USA. He has been an Associate Member of the Center for Clean Energy Engineering, UCONN, since 2009. He received several international paper awards including a best paper award at the IEEE Power Conversion Conference 2007, and an outstanding writing award in the International Future Energy Challenge, in 2007. He also received National Science Foundation (NSF) CAREER Award in 2015. His current research interests include smart and efficient energy and power conversion systems, renewable and distributed generation integration, smart buildings, and microgrid applications.

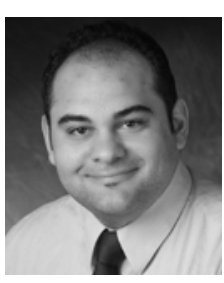

Ali M. Bazzi received his B.S. and M.S. degrees in Electrical Engineering from the American University of Beirut, Beirut, Lebanon, in 2006 and 2007, respectively, and his Ph.D. degree from the University of Illinois at Urbana-Champaign (UIUC), Urbana, IL, USA, in 2010. He is presently an Assistant Professor with the Department of Electrical and Computer Engineering, University of Connecticut (UCONN), Storrs, CT, USA, where he established and directs the Advanced Power Electronics and Electric Drives Laboratory. He was a Senior Power Electronics Electrical Engineer with Delphi Electronics \& Safety, Kokomo, IN, USA, in 2011-2012, and a Visiting Assistant Professor with UIUC, in Spring 2011. He was the recipient of the Mavis Memorial Scholarship at UIUC, in 2009, and the Outstanding Teaching Award from the Department of Electrical and Computer Engineering, UCONN, in 2014. His current research interests include the design, control, optimization, and reliability modeling of power electronics with applications in motor drives and solar photovoltaic systems, as well as renewable energy integration in micro-grids and the real-time control and optimization of energy systems. 